\title{
The effect of two types exercise therapy on bone mineral density and mechanical strength of osteoporotic male rats
}

\author{
Maryam Banparvari $^{1^{*}}$, Abbas Salehikia ${ }^{1}$, Fatemeh Azarm ${ }^{2}$
}

1. Department of Sport Sciences, Faculty of Education and Psychology, University of Sistan and Baluchestan, Zahedan, Iran

2. Master of sport Management, Ilam, Iran

*Corresponding author:Tel: +98 9902333592 Fax: +98 31132207

Address: Department of Sports Sciences, Faculty of Education and psychology, Sistan and Baluchestan University, Zahedan, Iran

E-mail: banparvari@ped.usb.ac.ir

Received; 2017/03/20 revised; 2017/04/9 accepted; 2017/05/20

\section{Abstract}

Introduction: Osteoporosis is a systemic skeletal disease with a consequent increase in bone fracture. The purpose of this study was to determine skeletal changes induced by two types of exercise therapy in osteoporotic rats.

Materials and methods: Among 30 male rats, 6 of them were selected as healthy group, Then Osteoporosis induced in other rats by intraperitoneal injection of $20 \%$ ethanol solution $(3 \mathrm{~g} / \mathrm{kg} / \mathrm{day})$ for 3 weeks. Osteoporotic rats divided into 4 groups: Baseline, resistance, endurance and control $(n=6)$. The two exercise groups completed 12 weeks of training, 5 days/week according to protocols. Endurance protocol included running exercise on a treadmill for, $12 \mathrm{~m} / \mathrm{min}, 10$ to $64 \mathrm{~min} /$ day. The Resistance training protocol consisted of 8 series of climbing on the $110 \mathrm{~cm}$ vertical ladder angled at $80^{\circ}$ with weights tied to animal tail that was increased from the $50 \%$ of the body mass of the animal in the first series to $100 \%$ in the eighth series. At the end of the training, the animals were euthanized, and the BMD of the femur and the L4 + L5 vertebrae were measured using DXA and tensile max load of the tibia and compression max load of the L5 were measured applying Zwick 2.5 machine. The collected data was analyzed using paired $t$ and one way ANOVA tests.

Results: The two groups of the exercises had significantly increased femur BMD compared to controls $(\mathrm{P}=0.035, \mathrm{P}=0.001)$. $\mathrm{L} 4+\mathrm{L} 5 \mathrm{BMD}$ in resistance training and control groups was significantly greater than endurance group $(\mathrm{P}=0.001, \mathrm{P}=0.001)$. The tibia tensile maximum load and L5 maximum load in resistance group were significantly greater than control $(\mathrm{P}=0.01, \mathrm{P}=0.03)$.

Conclusion: Resistance training induces more effective favorable changes in bone mineral status and bone strength as compared to endurance exercise in osteoporotic male rat.

Keywords: Osteoporosis, Exercise therapy, BMD, Bone mechanical strength

\section{Introduction}

Osteoporosis is a disease characterized by compromised bone mass and strength, resulting in an increase in bone fragility (1). 50 percent of men and 70 percent of women of up to 50 years old in Iran suffer from osteoporosis or osteopenia (2). Hip and vertebra compression fractures are the most prevalent fractures in osteoporotic patients which besides high medical expenses (7), have high mortality risk (3, 4). Secondary side effects of osteoporosis such as vertebral deformity due to the anterior vertebral compression can also result in symptoms like back pain and aspirational deficiency (4-6). While there are many pharmacological agents available for the prevention and treatment of OP, like Bisphosphonates, widespread and long 
term use of these agents, it is limited due to its side-effects, costs and poor long term compliance. Therefore, it is essential that non-pharmacological strategies prevent osteoporosis to continue to be evaluated (1). Evidences show that exercise therapy, without any side effect has mechanical as well as hormonal influences on bone structure and accumulation of mineral in regions of the bone undergoing loading. In addition, it strengthens muscles and improves balance, thus reduces the overall risk of falls and fractures (1). Although the biological processes in bone estrogenic reaction to mechanical loads are not completely known, yet findings reveal that these processes can be impaired with aging (8) and the bone formation can improve just by specific exercises. Despite numerous papers and researches regarding the effect of physical exercises on osteoporosis, many discrepant results have been reported. For example results of a study by Yoshinobu (2009) showed that 8 weeks running on treadmill (15 meters/ minutes, 5 days/ weeks, 30 minutes/day) can increase femurs BMD of osteoporotic male rats while the same protocol with 180 minutes duration decrease femur BMD (9). In another research conducted by Iyamoto (2009) in order to make a comparison among three different intensities of aerobic exercises, after 12 week of exercise, a significant increase in the tibia BMD and Mechanical strength of femur was observed in only EX1) 12 meters/minutes, 1 hour/day protocol) compared with that in group control, while any significant increase in the L4+L5 vertebral BMD was not observed in any exercise groups compared with that the control group. However, there was no significant difference in a maximum breaking force of the L5 vertebral body among all the exercise and control groups (10). Furthermore, Joo et al (2013) showed that 10 weeks exercise on treadmill can improve bone strength through increasing BMD, max load and cortical thickness (11). Accordingly, in order to compare the effects of 8 week endurance and resistance exercises,Aguiar (2010) study and Timo Hinrich et al (2010) showed that only resistance group increased the mechanical strength of femur; this study also showed different responses of two types of exercise therapy programs to mechanical features of osteoporotic rats' femurs $(12 \&$ 13). In contrast, the results of Rodrigues research (2013) showed the potential treatment effects of two endurance and resistance protocol on protecting bones from osteoporotic fractures (14). Despite the proving evidences of the positive effects of exercise therapy on bones in studies, the effective exercise principles on BMD such as frequency, duration and intensity have been not definitely confirmed (15). On the other hand, BMD as the most common method to assess bone is only one of the indices of bone health, while bone mechanical strength stems from the interaction of several different features. Moreover there are contradictory reports about the effect of exercise therapy on BMD and bone mechanical strength. In Lucas et al (2013) study, resistance exercise increase femur strength but no increase occurred in femur BMD (16). On the other side, due to the necessary non-invasive assessment of the human bone, these studies are focused on evaluation of effectiveness of exercise therapy on the (BMD) DEXA results. In the present study, a mechanical test which objectively measures bone resistance against breakage is applied beside DEXA; so that, in case of consistency of the both methods results, the result of DEXA can be implied with more certainty. Regarding the sensitivity of the weight bearing bones like femur to treadmill exercise, and also the topical exercises on waist, we investigated the site specific theory on the effect of exercise on bones. Thus the main purpose of the present study was comparison between the effect of two types of exercise therapies on femur and L4 and 5 BMD and Bone strength of Osteoporotic rats. 


\section{Materials and methods}

This study was a true experimental study. 30 Wistar mature male rats with mean weight of 180-200 g were purchased. Animals were housed 3 to a cage and kept in a room with a temperature of $22 \pm 2{ }^{\circ} \mathrm{C}$, relative humidity of $55 \pm 5 \%$ and a $12 \mathrm{~h}$ light and dark cycle. They were allowed free access to drinking water and they were fed by pellet feeding made by Karaj Behparvar Company. Their weight and received food were measured each day before exercises. 6 rats were killed in order to determine the normal bone parameters, then osteoporosis was induced experimentally in 24 other male rats by intraperitoneal injection of $20 \%$ alcohol ( 3 gr / kg / day) for 3 week (17). The osteoporotic rats, at the age of 12 weeks, were stratified by weight and randomly assigned to the 4 groups (6 rats in each group): baseline group, EX1 (endurance group), EX2 (resistance group) and Con (control group). The two exercise groups completed 12 weeks of training, 5 days a week according to protocols. Endurance protocol included running exercise on a flat - bed treadmill for, 12 meters/ minute, 5 days/week, 10 minutes/day in the first week and increasingly up to 64 minutes on the 12th week. The one-week running familiarization protocol was 8 to 10 meters/minutes, 5 days/week and 5 to 10 minutes/ day (18). The animals of the resistance group were subjected to a progressive strength exercise, 5 sessions per week, for 12 week. Each session consisted of 8 climbing series on the 110$\mathrm{cm}$ vertical ladder angled at $80^{\circ}$, with a progressively heavier load fixed to the proximal part of the animal tail with Tape. A housing chamber was located at the top of the ladder and served as a shelter during the resting period. For each series, the animals had to make 8-12 dynamic repetitive movements to reach the housing chamber. In the first two series, the load was $50 \%$ of the animal's total body mass; in subsequent series, the load was progressively increased to a final load of $100 \%$. The rest interval between the series was $60 \mathrm{~s}$. One week familiarization protocol with ladder was conducted before the main exercises that consisted of three trials per day for three days. In the first trial, the rats were kept in the housing chamber for $60 \mathrm{~s}$ and then placed on the ladder, $35 \mathrm{~cm}$ from the top. In the second and third trial, the rats were placed in the middle and bottom of the ladder. Twentyfour hours after the last training session, the animals were euthanized. Left tibia, right femur and L4+L5 were removed from surrounding tissue and kept in freezer in - $20 c^{\circ}$ temperature. Before the tests, the bones were thawed at room temperature and moisturized with saline femur metaphysis BMD and L4+L5 BMD $\left(\mathrm{gr} / \mathrm{cm}^{2}\right)$ were measured by DEXA (LEXXOS, USA) in a radiology center. After densitometry, L5 were kept in $-20^{\circ} \mathrm{C}$ temperature for mechanical test (11). The mechanical tests were performed in the biomechanics laboratory of the Tarbiat Modares University. The biomechanical properties of the tibia and L5 were determined using tensile and compression strength tests respectively in a universal testing machine (Zwick 2/5 HS Germany WN 150888). Each tibia was placed on a special holding device with two supports, located at a distance of $17 \mathrm{~mm}$. The tibias were loaded to failure in tensile test at the middiaphysis, with a speed of $0.1 \mathrm{~mm} /$ second. In the L5 compression test, by removing the cranial and caudal ends of the specimens, a flat cylinder shape was obtained for compression test. The vertebral cylinder Sample was placed centrally on the lower smooth surface of steel disk. The compression force was applied by another steel disk with $2 \mathrm{~mm} /$ minute speed. At the end of the mechanical tests, the values for maximal load $(\mathrm{N})$ were obtained from loaddeformation curve. SPSS 20 were used for statistical analysis. All value were expressed as mean and standard deviation. ANOVA test was used to compare the 
significant differences in BMD and bone mechanical strength among the exercises and control groups after 12 weeks of exercise. Paired t test was used to compare the significance differences in research variables, before and after the 12 weeks exercises. A significance level of $\mathrm{p}<0.05$ was used for all comparisons.

\section{Results}

There was no significant difference between the body weights of groups (Table 1). The result of the study showed that BMD and bone strength significantly decreased after the ethanol injection. As it is indicated at Table 2, 12 weeks endurance and resistance exercises caused significant increase in femur BMD compared with baseline values $(\mathrm{P}=0.001$, $\mathrm{P}=0.000)$ while, there was not such a significant increase in control group $(\mathrm{P}=0.144)$. The L4+L5 BMD of two control and resistance groups had significant increase compared with baseline values $(\mathrm{P}=0.000$ and $\mathrm{P}=0.002)$ while this difference was not significant in endurance group $(\mathrm{P}=0.235)$. Based on the ANOVA analysis, a significant increase in the femur BMD was observed in Resistance and endurance group compared with that in group control (respectively $\mathrm{P}=0.035$ and $\mathrm{P}=0.001$ ). In other hand, $\mathrm{a}$ significant increase in the L4+L5 BMD was observed in Resistance and control group compared with that in group endurance $(\mathrm{P}=0.001$ and $\mathrm{P}=0.001)$ while L4+L5 BMD of two control and resistance groups had no significant statistical difference $(\mathrm{P}=0.999)$.

Table 1. Physical parameters of the experimental rats.

\begin{tabular}{|c|c|c|c|c|c|c|}
\hline & \multicolumn{2}{|c|}{ Endurance $(n=7)$} & \multicolumn{2}{|c|}{ Resistance $(\mathrm{n}=7)$} & \multicolumn{2}{|c|}{ Control $(n=7)$} \\
\hline & Baseline & Post & Baseline & Post & Baseline & Post \\
\hline Age (week) & 12 & 24 & 12 & 24 & 12 & 24 \\
\hline Weight (gr) & $246.50 \pm 17.62$ & $393.50 \pm 16.59$ & $249.67 \pm 15.42$ & $404.17 \pm 12.36$ & $250.83 \pm 18.65$ & $408.67 \pm 8.82$ \\
\hline
\end{tabular}

All data represent mean \pm SD. Endurance, treadmill running exercised group. Resistance, progressive strength exercised group.

According to table 3 , a significant increase in L5 compression max load was only observed in Resistance and endurance group compared with that baseline values $(\mathrm{P}=0.001$ and $\mathrm{P}=0.005)$. The Tibial tensile max load result showed that none of the endurance, resistance and control group had any significant increase compared with that baseline values $(\mathrm{P}=0.44, \mathrm{P}=0.10$ and $\mathrm{P}=0.39$ ). Based on the ANOVA analysis, only L5 compression max load $(\mathrm{P}=0.03)$ and Tibial tensile max load $(\mathrm{P}=0.01)$ of resistance group were significantly higher than control group.

Table 2. The Femur and L4+L5 BMD, before and after 12-week exercise.

\begin{tabular}{lcccccc}
\hline & \multicolumn{2}{c}{ Endurance $(\mathrm{n}=7)$} & \multicolumn{2}{c}{ Resistance $(\mathrm{n}=7)$} & \multicolumn{2}{c}{ Control $(\mathrm{n}=7)$} \\
\cline { 2 - 7 } Femur & Baseline & Post & Baseline & Post & Baseline & Post \\
\cline { 2 - 7 } L4+L5 & $0.12 \pm 0.019$ & $0.19 \pm 0.022$ & $0.12 \pm 0.019$ & $0.21 \pm 0.006$ & $0.12 \pm 0.019$ & $0.15 \pm 0.032$ \\
\cline { 2 - 7 } & $0.08 \pm 0.018$ & $0.10 \pm 0.047$ & $0.08 \pm 0.018$ & $0.26 \pm 0.06$ & $0.08 \pm 0.018$ & $0.26 \pm 0.014$ \\
\hline
\end{tabular}

All data represent mean \pm SD. BMD, body mass index.

Table 3. Mechanical properties in the Tibia mid-diaphysis and L5, before and after 12-week exercise.

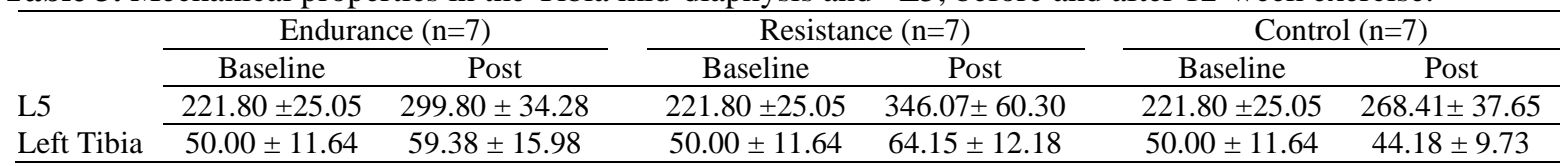

All data represent mean \pm SD. 


\section{Discussion}

After 12 weeks, the femur BMD of endurance group increased in compare with control group. Moreover, L5 compression, Tibial tensile max load and femur BMD of resistance group were significantly higher than control group. It shows that exercise can result in positive skeleton balance of animal, although the specific mechanisms by which exercise influences bone metabolism are still not thoroughly understood (20). Exercise is considered to play a crucial role in bone modeling and remodeling. Physical activity is an important lifestyle factor that has the potential to modify bone strength through its effects on bone mineral density and geometry $(1,21,22)$. Our results indicated that the femur BMD and tibial tensile max load of indurance group, were higher than control group but this increase was only significant in case of femur BMD. The effect of endurance exercise on increasing BMD of weigh bearing bones like femur has been reported by several studies $(20,14)$. These results of the present study are consistent and agree with Canus (23) and Moskild (24) who respectively showed the effects of 8 weeks and 4 months running on treadmill on increasing Femur BMD of rats. Furthermore, the present results are consistent with Iwamoto (2009) and Joo (2013) findings, regarding the increase of femur BMD and the no increase in L4+L5 BMD. In contrast, the result of Borin (2010) study regarding the destructive effect of 11 weeks running on the treadmill with high intensity on skeleton system (25) and also Yoshinobu results show decrease in bone tissue of long bone due to 180 minute exercise on treadmill are not consistent with our study. Shimegi stated that intensity and duration of exercise are effective on increasing BMD. The homogeneity of the duration of exercise programs with the present study can confirm the consistency of the results. The running exercise of moderate intensity with a proper duration at which a little fatigue is observed in the exercise muscles, is effective to increase bone mass and mechanical strength in the weight-bearing long bones. However, probably the inconsistency of some studies' result with the present study's might be due to the difference in intensity, duration and mechanical load of exercises which are important in bones remodeling stimulation. There exists thresholds and optimal levels in both the intensity and duration of the exercise to improve the bones' quality, while increasing these exercise principles higher than the standard level can have negative effects on bones' quality (11). The local bone formation under the mechanical load, can justify the significant increase of femur BMD in endurance group compare with control. The result of this study showed that the femur BMD of endurance group significantly increase compare with control group while tibia max load, despite its increase compare with control group, was not significant. The differences in the bone tissue of the tibia, diaphysis and femur metaphysis can probably justify the difference results in the two bone areas. Studies have shown that, high blood flow of metaphyseal spongy tissue causes increasing the metabolic activities of this tissue and also its mechanical loading; thus this tissue is more effected by physical exercises rather than the cortical diaphysis tissue (9). Another part of the results reveal that L5 compression max load of endurance group has had no significant difference with control group and L4+L5 BMD has had a significant decrease compare to the two resistance and control group. Tibia and femur bones in rat are different from vertebrae, regarding their position in the body; thus during the exercise on treadmill, most of the mechanical load is imposed on them rather than the vertebrae, the results show that The adaptive response of bone to exercise differs between regions (11) and findings 
suggest that bone adaptation to treadmill exercise is site specific (1). Their results showed that L5 compression and Tibial tensile max load and BMD femur of resistance group were significantly higher than control group. It is clear that the weight bearing exercises increase the BMD in human and animals. These findings are consistent and agree with many previous studies in this area (12). Our findings are consistent with Romeo (2013) results, with regard to the increase in BMD femur, also with Notomi (26) who showed that the resistance exercise can increase periosteal bone formation parameters of femur of rats. Specialized researches show that exercise can probably increase tibial tensile strength through improving the collagen network organization and biomaterial bone properties (27). There are many probabilities regarding the effects of resistance exercise on increasing the bone mechanical strength and BMD which include: decreasing bone resorption by reducing the number of the osteoclasts and increasing bone formation by increasing osteoclasts' number, optimized mechanical load in resistance exercises, high strain rates and unusual strain distributions (1). The significant increase in L5 max load of resistance group, confirms the site specific theory of the exercise effect on waist. local exercise was applied on rats' waist by attaching a weight to animal's tail which resulting mechanical loads on the lumbar and results in strain of lumber and stimulation of the bone to respond to these new needs by new shaping, thus L5 max load would increase. It seems that these exercises in human, especially on the core area, besides reducing the Spinal curvature deformity by improving the bones' strength, the balance can also be improved through strengthen the core stability and prevent falling and fractures $(5,6)$. The results of the resistance exercises on tibia diaphysis and vertebrae show that this exercise can be effective on both types of cortical and trabecular tissues by optimized mechanical loading (27) and resistance exercise can probably effect on both bones' tissue. Another finding showed an inconsistency between the result of BMD and L5 load max of resistance group which reveals the necessity to consider different bone's assessments. Despite that BMD is the gold standard for diagnosis osteoporosis, this measure is only able to provide insights regarding the quantity of bone tissue. (21). However the Limitations of study design and use of non-invasive technologies in human, inevitably leads to the use of DEXA.

\section{Limitations}

Although the present study has offered some answers to some questions regarding bones' parameters, yet there are still many unknown ones. Besides, considering the different results of the mechanical assessment test (invasive) and DEXA (non-invasive), it should be mentioned that an extended volume of the present knowledge about the effect of exercise therapy on bone have been obtained via DEXA method, yet its limitations should be studied (14).

\section{Conclusion}

The results of two exercise therapy programs reveals that endurance exercises, only increase the BMD of long weigh bearing bones of osteoporotic rats, but the resistance exercise can lead to effective changes in BMD and bones' mechanical strength of osteoporotic rats. Thus it is probably possible to apply this exercise therapy in order to improve bones strength of osteoporotic people. 


\section{References}

1. Mohammed H, Saiem Aldahr. Bone Mineral Status Response to Aerobic Versus Resistance Exercise Training in Postmenopausal Women. World Appl Sci J. 2012; 16(6): 806-13.

2. Khorsandi M, Shamsi M, Jahani F. [The Survey of Practice About Prevention of osteoporosis Based on Health Belief Model in Pregnant Women in Arak City.] J Rafsanjan Univ Med Scie. 2013; 12(1): 35-46. (Persian)

3. Puisto $\mathrm{V}$, Heliövaara M, Impivaara $\mathrm{O}$, Jalanko T, Kröger H, Knekt P, et al. Severity of vertebral fracture and risk of hip fracture: a nested case-control study. Osteoporos Int. 2011; 22(1):63-8.

4. Boonen S, Wahl DA, Nauroy L, Brandi ML, Bouxsein ML, Goldhahn $\mathrm{J}$, et al. Balloon kyphoplasty and vertebroplasty in the management of vertebral compression fractures. Osteoporos Int. 2011; 22(12):291534.

5. Granito RN, Aveiro MC, Renno AC, Oishi J, Driusso P. Comparison of thoracic kyphosis degree, trunk muscle strength and joint position sense among healthy and osteoporotic elderly women: a cross-sectional preliminary study. Arch Gerontol Geriatr. 2012; 54(2): 199-202.

6. Wang Y, Owoc JS, Boyd SK, Videman T, Battié MC. Regional variations in trabecular architecture of the lumbar vertebra: associations with age, disc degeneration and disc space narrowing. Bone. 2013; 56(2):249-54.

7. Armas LA, Recker RR. Pathophysiology of osteoporosis: new mechanistic insights. Endocrinol Metab Clin North Am. 2012; 41(3):475-86.

8. Chen H, Zhou X, Fujita H, Onozuka $\mathrm{M}$, Kubo KY. Age-related changes in trabecular and cortical bone microstructure. Int $\mathrm{J}$ Endocrinol. 2013; 2013:213234.

9. Hagihara Y, Nakajima A, Fukuda S, Goto S, Iida $\mathrm{H}$, Yamazaki $\mathrm{M}$. Running exercise for short duration increases bone mineral density of loaded long bones in young growing rats. Tohoku J Exp Med. 2009; 219(2):139-43.

10. Iwamoto J, Yeh JK, Aloia JF. Differential effect of treadmill exercise on three cancellous bone sites in the young growing rat. Bone. 1999; 24(3):163-9.

11. Joo YI, Sone T, Fukunaga M, Lim SG, Onodera S. Effects of endurance exercise on three-dimensional trabecular bone microarchitecture in young growing rats. Bone. 2003; 33 (4):485-93.

12. Aguiar AF, Agati LB, Muller SS, Pereira OC, Dal Pai Silva M. Effects of physical training on the mechanical resistance of rat femur proximal thirds. Acta ortop. Bras. 2010; 18(5):245-9.

13. Hinrichs T, Chae EH, Lehmann R, Allolio B, Platen P. Bone Mineral Density in Athletes of Different Disciplines: a Cross Sectional Study. Sports Sci J. 2010; 3: 129-33.

14. De Souza RR, Do Carmo Sitta M, Santarem Sobrinho JM, Jacob Filho W. Long Term Running Exercise vs. Long Term Strength Exercise on Femoral Bone Mass Assessed in a Rat Model. J Exerc Physiol Online. 2013; 16(2): 92-8.

15. Colletti LA, Edwards J, Gordon L, Shary J, Bell NH. The effects of muscle-building exercise on bone mineral density of the radius, spine, and hip in young men. Calcif Tissue Int. $1989 ; 45(1): 12-4$.

16. Drummond LR, Del carlo RJ, Del C, Soares Melo SF, Carneiro-Junior MA, $\mathrm{Da}$ silva KA, et all .Enhanced femoral neck strength in response to 
weightlifting exercise training in maturing male rats. Int Sport Med J. 2013; 14(3):155-67.

17. El-Shenawy SMA, Yassin NAZ, A Badary O, EL-Moneem MA, ALShafeiy HM. Study of the effect of Allium porrum on osteoporosis induced in rats. Scholars Research Library. 2013; 5 (1):188-98.

18. Iwamoto J, Takeda T, Sato Y. Effect of treadmill exercise on bone mass in female rats. Exp Anim. 2005; 54(1):1-6.

19. Cassilhas, RC, Reis IT, Venâncio D, Fernandes J, Tufik S, Mello MTD. Animal model for progressive resistance exercise:a detailed description of model and its implications for basic research in exercise. Motriz Rio Claro. 2013; 19(1):178-84.

20. Maïmoun L, Sultan C. Effects of physical activity on bone remodeling Metabolism. 2011; 60(3):373-88.

21. Fonseca H, Moreira-Gonçalves D, Coriolano HJ, Duarte JA. Bone Quality: The Determinants of Bone Strength and Fragility. Sports Med. 2014; 44(1):37-53.

22. Allison SJ, Folland JP, Rennie WJ, Summers GD, Brooke-Wavell K. High impact exercise increased femoral neck bone mineral density in older men: A randomised unilateral intervention. Bone. 2013; 53 (2):3218.

23. Kannus P, Sievänen H, Järvinen TL, Järvinen $\mathrm{M}$, Kvist $\mathrm{M}$, Oja $\mathrm{P}$, et al. Effects of free mobilization and lowto high-intensity treadmill running on the immobilization-induced bone loss in rats. J Bone Miner Res. 1994; 9(10):1613-9.

24. Mosekilde L, Danielsen CC, Søgaard $\mathrm{CH}$, Thorling E. The effect of longterm exercise on vertebral and femoral bone mass, dimensions, and strength - assessed in a rat model. Bone. 1994; 15(3):293-301.

25. Bourrin S, Palle S, Pupier R, Vico L, Alexandre C. Effect of physical training on bone adaptation in three zones of the rat tibia, $\mathrm{J}$ Bone Miner Res. 1995 ;10(11):1745-52.

26. Notomi T, Okazaki Y, Okimoto N, Tanaka Y, Nakamura T, Suzuki M. Effects of tower climbing exercise on bone mass, strength, and turnover in growing rats. J Appl Physiol. 2002; 93 (3):1152-8.

27. Golub EE. Role of matrix vesicles in biomineralization. Biochim Biophys Acta. 2009; 7(12):1592-8. 